\title{
Fatores psicossociais associados ao desenvolvimento de transtornos psiquiátricos em crianças e adolescentes
}

\section{Psychosocial factors associated with the development disorders of psychiatric in teens}

Izabel Ester Inácio Ferraz ${ }^{1}$. Álvaro Jorge Madeiro Leite². Eugênio de Moura Campos ${ }^{3}$. Iago Farias Jorge ${ }^{4}$ Sarah Rodrigues do Espírito Santo ${ }^{4}$. Gabriel Albuquerque Parente ${ }^{4}$. Breno Milhomens Arraes ${ }^{4}$.

1 Psiquiatra do Hospital Universitário Walter Cantídio (HUWC), Universidade Federal do Ceará (UFC), Fortaleza, Ceará, Brasil. 2 Doutor em pediatria pela Universidade Federal de São Paulo (UNIFESP), Professor Titular do Departamento de Pediatria da Universidade Federal do Ceará (UFC), Fortaleza, Ceará, Brasil. 3 Doutor em Farmacologia, Professor Titular do Departamento de Medicina Clínica da Universidade Federal do Ceará (UFC), Fortaleza, Ceará, Brasil. 4 Aluno do Curso de Medicina da Universidade Federal do Ceará (UFC), Fortaleza, Ceará, Brasil.

\section{RESUMO}

Introdução: o objetivo deste estudo foi avaliar a prevalência de comportamentos e sintomas relacionados ao desenvolvimento de transtornos psiquiátricos em crianças e adolescentes em uma população de 307 crianças acompanhadas em ambulatórios de pediatria do Hospital Infantil Albert Sabin (HIAS), em Fortaleza, Ceará. Métodos: o Questionário de Capacidades e Dificuldades (SDQ) foi o instrumento principal do estudo, além de um formulário sociodemográfico desenvolvido pelos autores, contendo possíveis fatores associados ao desenvolvimeto de transtornos mentais. O SDQ possui 25 questões que compõem cinco subgrupos de sintomas psiquiátricos. Resultados: encontramos percentuais elevados de crianças com comportamento pró-social não normal $(10,1 \%)$, hiperatividade $(30,3 \%)$, sintomas emocionais $(61,6 \%)$, problemas de conduta $(45,9 \%)$ e problemas de relacionamento com colegas $(32,9 \%)$. A análise univariada revelou os seguintes fatores como estatisticamente significativos $(p<0,05)$ para $o$ surgimento potencial de transtornos mentais: doença mental dos pais, depressão materna no período até 03 meses após o nascimento da criança e pais que não vivem juntos atualmente. Discussão: a prevalência de transtornos mentais em crianças e adolescentes acompanhados por outras comorbidades clínicas é consideravelmente maior que na população geral; e os fatores significativos para o desenvolvimento de transtornos mentais na criança estão relacionados aos pais.

Palavras-chave: Crianças. Adolescentes. Psiquiatria. Comportamento social. Transtornos mentais.

\section{ABSTRACT}

Introduction: the aim of this study was to evaluate the prevalence of behaviors and symptoms related to the development of psychiatric disorders in children and adolescents in a population of 307 children in a pediatric outpatient clinic at Hospital Infantil Albert Sabin (HIAS) in Fortaleza, Ceará. Methods: the Strengths and Difficulties Questionnaire (SDQ) was the main tool of the study, and a sociodemographic form developed by the authors, containing possible factors associated with the development of mental disorders. The SDQ has 25 questions that make up five subgroups of psychiatric symptoms. Results: we found high percentage of children with no normal social pro behavior (10.1\%), hyperactivity $(30.3 \%)$, emotional symptoms (61.6\%), conduct problems $(45.9 \%)$ and relationship problems with colleagues $(32.9 \%)$. Univariate analysis revealed the following factors as statistically significant $(\mathrm{p}<0.05)$ for the potential emergence of mental disorders: mental illness of parents, maternal depression in the period up to 03 months after the child's birth and parents that are not currently living together. Discussion: the prevalence of mental disorders in children and adolescents accompanied by other medical comorbidity is considerably higher than in the general population; and the significant factors for the development of mental disorders in children are related to parents.

Keywords: Child. Adolescent. Psychiatry. Social behavior. Mental disorders.

Autor correspondente: Izabel Ester Inácio Ferraz, Rua Antônio Augusto, 1404, Meireles, Fortaleza, Ceará. Telefone: +55 85 3231-8031/ 996766207. E-mail: izabeleiferraz@gmail.com

Conflito de interesses: Não há qualquer conflito de interesses por parte de qualquer um dos autores.

Recebido em: 15 Mai 2016; Revisado em: 22 Jul 2016; Aceito em: 29 Ago 2016. 


\section{INTRODUÇÃO}

Os transtornos psiquiátricos em crianças e adolescentes são condições prevalentes, precoces e persistentes ao longo da vida, com impacto sobre o funcionamento e o desenvolvimento do indivíduo, e pesado ônus sobre a família e a sociedade. ${ }^{1}$

Há uma demanda no mundo todo por psiquiatras da infância e adolescência. No Brasil, a distribuição destes profissionais é mais precária, com maior concentração nos centros urbanos. Cada vez mais, pediatras, hebiatras e clínicos tratam esses pacientes na ausência do psiquiatra infantil. São necessários mais estudos para um melhor conhecimento dos transtornos psicopatológicos da infância e da adolescência, para permitir a implantação de programas de prevenção e intervenção precoces. $^{2}$

Waddell et $\mathrm{al}^{1}$ revisaram a epidemiologia dos transtornos mentais na população pediátrica no Canadá, Estados Unidos, Grã-Bretanha, Austrália e Nova Zelândia. ${ }^{3}$ A prevalência global destes transtornos foi estimada em $14,3 \%$, sendo mais frequentes transtornos de ansiedade $(6,4 \%)$, transtorno de déficit de atenção/hiperatividade (TDAH; 4,8\%), transtorno de conduta $(4,2 \%)$ e transtornos depressivos (3,5\%). As estimativas no Brasil são um pouco maiores do que em países desenvolvidos, mas, geralmente, correspondem à literatura internacional. ${ }^{1}$

As influências sobre o desenvolvimento da criança são múltiplas e complexas, e sobrepõem-se de maneira ainda pouco compreendida. ${ }^{4}$ Existem fatores biológicos, como genética (psicopatologia parental), exposições a toxinas, complicações da gravidez e do parto, e fatores psicológicos, como traços disfuncionais de personalidade e transtornos de aprendizagem; principalmente nos países em desenvolvimento, destacam-se os fatores sociais e familiares, como práticas disfuncionais de parentalidade (falta de supervisão ou regras, rejeição aos filhos, estilo disciplinar punitivo), violência entre pais, separação conjugal, pais criminosos, família muito numerosa ou de baixa renda. Entre os fatores escolares e comunitários destacam-se: influência de pares desviantes, escola com alta taxa de delinquência e vizinhança com alta criminalidade. ${ }^{5}$

Individualmente, contudo, os fatores de risco têm valor preditivo limitado. Nem sempre vida urbana, colapso do casamento, criminalidade dos pais, marginalidade social ou falta de escola estarão relacionados a crianças com transtornos de conduta. Sabemos que crianças e adolescentes podem sofrer influência desses fatores no decorrer de suas vidas e se tornar adultos com boas habilidades pessoais e profissionais. Diversos fatores e suas inter-relações devem ser considerados, pois sinais e sintomas mudam com a idade e com o tempo. ${ }^{6}$

Esta investigação baseia-se na suposição de que crianças e adolescentes acompanhados nas subespecialidades pediátricas estão sobrecarregados pela morbidade que as conduz ao ambulatório, e podem ser portadores de morbidade psiquiátrica oculta.

O objetivo desse estudo foi descrever a prevalência de comportamentos e sintomas relacionados ao desenvolvimento de transtornos psiquiátricos em crianças e adolescentes assistidos em ambulatórios de especialidades pediátricas, e fatores associados a esses transtornos.

\section{MÉTODOS}

Trata-se de um estudo transversal, de base hospitalar (Hospital Infantil Albert Sabin (HIAS)), realizado no município de Fortaleza, nos ambulatórios de especialidades pediátricas. A população do estudo foi constituída por 307 crianças e adolescentes, com idade entre 7 e 17 anos, de ambos os sexos. Os serviços de Psiquiatria e Neuropediatria não foram incluídos por já acompanharem crianças e adolescentes com transtornos psiquiátricos, e nosso estudo utilizar um instrumento de triagem para esses transtornos. O instrumento utilizado foi o Strengths and Difficulties Questionnaire (SDQ, Questionário de Capacidades e Dificuldades), construído em 1997 por Goodman e validado no Brasil em 2000 por Fleitlich, Cartázar e Goodman, na versão para pais. A coleta de dados foi realizada em agosto e setembro de 2015. O SDQ possui 25 questões sobre capacidades e dificuldades, divididas em cinco subgrupos de sintomas psiquiátricos: sintomas emocionais, de conduta, hiperatividade, problemas de relacionamento com colegas e comportamento pró-social. ${ }^{7}$

Cada escala é composta de 5 itens, que podem ser pontuados como mais ou menos verdadeiro (calculado como 1), ou falso ou verdadeiro (variando conforme o item, podendo pontuar 0 ou 2). A pontuação de cada escala pode variar de 0 a 10 se os 5 itens forem completados. O resultado de cada escala pode ser avaliado se ao menos 3 itens forem completados. ${ }^{7}$

A pontuação total de dificuldades é gerada pela soma dos resultados de todas as escalas, exceto a de sociabilidade. O resultado pode variar de 0 a 40 . No Quadro 1, estão as pontuações de cada escala e a total, dividindo os grupos em normal, limítrofe e anormal. ${ }^{7}$

Quadro 1. Pontuações das subescalas do SDQ.

\begin{tabular}{|l|l|l|l|}
\hline & Normal & Limítrofe & Anormal \\
\hline $\begin{array}{l}\text { Pontuação total das } \\
\text { dificuldades }\end{array}$ & $0-13$ & $14-16$ & $17-40$ \\
\hline $\begin{array}{l}\text { Pontuação dos sintomas } \\
\text { emocionais }\end{array}$ & $0-3$ & 4 & $5-10$ \\
\hline $\begin{array}{l}\text { Pontuação de problemas de } \\
\text { conduta }\end{array}$ & $0-2$ & 3 & $4-10$ \\
\hline $\begin{array}{l}\text { Pontuação para } \\
\text { hiperatividade }\end{array}$ & $0-5$ & 6 & $7-10$ \\
\hline $\begin{array}{l}\text { Pontuação para problemas } \\
\text { com colegas }\end{array}$ & $0-2$ & 3 & $4-10$ \\
\hline $\begin{array}{l}\text { Pontuação para } \\
\text { comportamento pró-social }\end{array}$ & $6-10$ & 5 & $0-4$ \\
\hline
\end{tabular}

Fonte: Goodman R. Questionário de Capacidades e Dificuldades(SDQ). Disponível em: http://www.sdqinfo.com/py/ sdqinfo/b3.py?language $=$ Portugueseqz $($ Brazil $)$ 
Em um cenário em que a amostra seja de alto risco, os "casos" podem ser identificados pela pontuação anormal ou limítrofe em uma das quatro escalas de dificuldades; quando a amostra é de baixo risco, os "casos" podem ser identificados pela pontuação anormal em uma das quatro escalas de dificuldades. Consideramos nossa amostra de alto risco, por serem crianças com comorbidades clínicas, portanto, chamamos o grupo "limítrofe+anormal" de "casos".

A variável dependente é definida como "casos". Utilizamos, além do SDQ, um formulário elaborado pelos autores, com questões sobre variáveis demográficas (cor, idade, escolaridade, situação conjugal dos pais, renda), história obstétrica da mãe (se planejou/desejou a gestação, uso de medicações/drogas, depressão gestacional ou 03 meses pós-parto), dados da criança (atividades escolares, de lazer; sono, história patológica), história familiar de doença mental e variáveis sociais (adoção, moradores da casa, abuso sexual, métodos educativos violentos). Estas são variáveis independentes.

Os dados foram organizados em formato padrão, com entrada de dados feita no aplicativo EPI INFO, versão 3.5.1. As medidas de associações foram baseadas em razão de prevalência (RP), com intervalo de confiança de 95\% (IC95\%). Na análise univariada, investigou-se isoladamente a relação entre cada variável explicativa e a variável dependente (Capacidades e Dificuldades).

A entrevista foi conduzida pela pesquisadora principal e alunos da Faculdade de Medicina da Universidade Federal do Ceará, componentes da Liga do Projeto de Vivência da Relação Médico Paciente e da Liga de Neurologia e Psiquiatria, treinados através de 6 seminários teóricos sobre os instrumentos e aplicação prática, por cada aluno, em dois pacientes para teste e retirada de dúvidas.

O estudo foi aprovado pelo Comitê de Ética do Hospital Infantil Albert Sabin (número 1.154.890), de acordo com os preceitos contidos na Declaração de Helsinki. O protocolo de pesquisa teve anuência da diretora clínica do HIAS Patrícia Jereissati Sampaio e da coordenadora do Ambulatório de Especialidades Sônia Maria Santos Guerreiro. A participação anônima e voluntária foi assegurada, pelo Consentimento Livre e Esclarecido, antes da realização das entrevistas.

\section{RESULTADOS}

Do total da amostra (307), 135 crianças foram consideradas casos (44\%). Encontramos amostras equivalentes de crianças do sexo masculino e feminino, de nacionalidade brasileira (exceto dois estrangeiros). A maior parte das crianças nasceu e mora em Fortaleza e tem cor parda.

Como mostrado na Tabela 1, a maioria das mães terminou o ensino médio e a maioria dos pais estudou até o ensino fundamental incompleto. Pais e mães, em sua maioria, têm idade entre 31 e 40 anos. Quase 50\% das mães trabalhavam e $50 \%$ estavam desempregadas, enquanto $80 \%$ dos pais encontravam-se trabalhando. A maioria das mães e pais eram casados ou tinham união estável.
Tabela 1. Perfil sociodemográfico dos pais das crianças e adolescentes.

\begin{tabular}{lllll}
\hline Variáveis & Pais & \multicolumn{3}{c}{ Mães } \\
Idade(anos) & $\mathbf{N}^{\mathbf{0}}$ & $\mathbf{\%}$ & $\mathrm{N}^{\mathbf{0}}$ & $\mathbf{\%}$ \\
Não sabe responder & 15 & 4,9 & 4 & 1,3 \\
$<21$ & 0 & 0 & 1 & 0,3 \\
21 -30 & 26 & 8,5 & 60 & 19,6 \\
31 -40 & 137 & 44,6 & 154 & 50,2 \\
$>40$ & 129 & 42 & 88 & 28,6 \\
Escolaridade & & & & \\
Não sabe responder & 22 & 7,2 & 5 & 1,6 \\
Analfabeto & 18 & 5,9 & 9 & 2,9 \\
Fundamental incompleto & 101 & 32,9 & 78 & 25,4 \\
Fundamental completo & 39 & 12,7 & 38 & 12,4 \\
Médio incompleto & 24 & 7,9 & 47 & 15,3 \\
Médio completo & 95 & 30,9 & 104 & 33,9 \\
Superior incompleto & 2 & 0,6 & 9 & 2,9 \\
Superior completo & 6 & 1,9 & 17 & 5,6 \\
Situação conjugal & & & & \\
Não sabe responder & 34 & 11 & 0 & 0 \\
Solteiro & 54 & 17,6 & 91 & 29,8 \\
Casado/união consensual & 219 & 71,4 & 214 & 70,2 \\
Trabalho & & & & \\
Não sabe responder & 23 & 7,5 & 3 & 0,97 \\
Empregado & 244 & 79,5 & 154 & 50,1 \\
Não empregado & 38 & 12,4 & 150 & 48,9 \\
\hline & & & & \\
\hline
\end{tabular}

Como mostrado na Tabela 2, a maioria das crianças frequenta escola pública, possui rendimento escolar na média e não frequenta reforço. A maioria não pratica esportes, lê livros de histórias, vê filmes, tem eletrônicos no quarto; tem atividades de lazer com a família, frequenta algum tipo de cerimônia religiosa, não compartilha quarto com os pais, e nunca se submeteu a tratamentos psicológicos prévios. Sobre histórico familiar, 36 crianças $(11,7 \%)$ têm um dos pais com doença mental e 264 (86\%) não têm pai ou mãe com doença mental.

Sobre história obstétrica das mães biológicas, a maioria não sofreu nenhum aborto, não usou medicações durante a gestação, nem outras substâncias, e não teve depressão durante a gestação nem nos três meses após o parto. Esses dados estão apresentados na Tabela 3 .

O Questionário de Capacidades e Dificuldades (SDQ) classifica as crianças em Normais e Casos (pontuação "limítrofe"+"anormal"). Como explanados na Tabela 4, foram considerados casos um número de 135 crianças (44\%), e encontramos prevalência de $30,3 \%$ para hiperatividade, 
$61,6 \%$ para sintomas emocionais, $45,9 \%$ para problemas de conduta, $32,9 \%$ para problemas de relacionamento e $10,1 \%$ para a escala de comportamento pró social não normal.

Tabela 2. Distribuição das crianças e adolescentes segundo perfil demográfico, rotina diária e histórico familiar de doença mental.

\begin{tabular}{lll}
\hline Variáveis & $N^{0}$ & $\%$ \\
\hline Sexo &
\end{tabular}

\section{Sexo}

Masculino

156

50,8

Feminino

49,2

Cor

Não sabe responder

Branca

1

105

0,3

Negra

Parda

10

190

Amarela

1

Local de moradia

Fortaleza

Interior

Frequenta escola

$$
\text { Sim }
$$

Não

Tipo de escola

Não sabe responder

Pública

Privada

Rendimento escolar

Não sabe responder

Acima da média

Na média

168

Abaixo da média

Defazagem severa

Frequenta reforço escolar

$\begin{array}{lll}\text { Não sabe responder } & 3 & 1,0 \\ \text { Sim } & 82 & 26,7 \\ \text { Não } & 222 & 72,3\end{array}$

\section{Pratica esporte}

$\begin{array}{lll}\text { Não sabe responder } & 1 & 0,3 \\ \text { Sim } & 94 & 30,6 \\ \text { Não } & 212 & 69,1\end{array}$

Lê livros de histórias

Não sabe responder

2
Continuação

\begin{tabular}{lll}
\hline Variáveis & $\mathbf{N}^{\mathbf{0}}$ & $\mathbf{\%}$ \\
\hline Sim & 201 & 65,4 \\
Não & 104 & 33,9
\end{tabular}

\section{Assiste a filmes}

$\begin{array}{lll}\text { Não sabe responder } & 1 & 0,3 \\ \text { Sim } & 179 & 58,3 \\ \text { Não } & 127 & 41,4\end{array}$

Tem eletrônicos no quarto

$\begin{array}{lll}\text { Não sabe responder } & 9 & 2,9 \\ \text { Sim } & 222 & 72,3 \\ \text { Não } & 76 & 24,8\end{array}$

Atividades de lazer com a família

Não sabe responder

Sim

Não

Frequenta cerimônia religiosa

$\begin{array}{lcc}\text { Sim } & 252 & 82,1 \\ \text { Não } & 55 & 17,9\end{array}$

Pais vivem juntos

Sim $176 \quad 57,3$

Não

Compartilha quarto com pais

Sim

Não

Tratamentos psicológicos prévios

Não sabe responder

Sim

Não

Doença mental em um dos pais

Não sabe responder

Sim

Não

86

Tabela 3. História obstétrica materna.

\begin{tabular}{lll}
\hline Variáveis & $\mathbf{N}^{\mathbf{0}}$ & $\mathbf{\%}$ \\
\hline Mãe realizou aborto $(\mathbf{n}=\mathbf{3 0 4})$ & 78 & 25,7 \\
Sim & 226 & 74,3 \\
Não & & \\
Usou medicações durante gestação $(\mathbf{n}=\mathbf{2 9 9})$ & & \\
\hline
\end{tabular}

Continua 
Continuação

\begin{tabular}{lll}
\hline Variáveis & $\mathbf{N}^{\mathbf{0}}$ & $\mathbf{\%}$ \\
\hline Sim & 48 & 16,1 \\
Não & 251 & 83,9 \\
Usou substâncias durante gestação (n=302) & & \\
Sim & 24 & 7,9 \\
Não & 278 & 92,1 \\
Gestação planejada (n=302) & & \\
Sim & 124 & 41,1 \\
Não & 178 & 58,9 \\
Gestação desejada (n=305) & & \\
Sim & 268 & 87,9 \\
Não & 37 & 12,1 \\
Depressão materna durante gestação (n=304) & & \\
Sim & 18 & 5,9 \\
Não & 286 & 94,1 \\
Depressão materna até 3 meses após gestação & \\
(n=304) & & \\
Sim & 18 & 5,9 \\
Não & 286 & 94,1 \\
\hline
\end{tabular}

Tabela 4. Distribuição das crianças e adolescentes segundo classificação do SDQ.

\begin{tabular}{lll}
\hline Pontuação total & N & \% \\
\hline Normal & 172 & 56,0 \\
Limítrofe & 51 & 16,6 \\
Anormal & 84 & 27,4 \\
Limítrofe+anormal=casos & 135 & 44,0 \\
Escala de comportamento pró-social & & \\
Normal & 276 & 89,9 \\
Casos & 31 & 10,1 \\
Escala de hiperatividade & & \\
Normal & 214 & 69,7 \\
Casos & 93 & 30,3 \\
Escala de sintomas emocionais & & \\
Normal & 118 & 38,4 \\
Casos & 189 & 61,6 \\
Escala de problemas de conduta & & \\
Normal & 166 & 54,1 \\
Casos & 141 & 45,9 \\
Escala de problemas de relacionamento com os & & \\
colegas & & \\
Normal & 206 & 67,1 \\
Casos & 101 & 32,9 \\
\hline
\end{tabular}

Em relação aos fatores psicossociais e à prevalência desses transtornos mentais, a análise bivariada encontrou associação estatisticamente significativa $(p<0,05)$ para as seguintes variáveis: depressão materna no período até 03 meses após o nascimento da criança $(\mathrm{p}=0,04)$, doença mental dos pais $(\mathrm{p}=0,04)$ e pais que não vivem juntos atualmente $(\mathrm{p}=0,0002)$.

\section{DISCUSSÃO}

Transtornos psiquiátricos da infância e adolescência no Brasil são frequentes. Em 2008, a Associação Brasileira de Psiquiatria estimou em 5 milhões o número de pessoas entre 6 e 17 anos com sintomatologias importantes a ponto de necessitar de tratamento em saúde mental. Este estudo encontrou percentuais bem mais elevados de crianças com potenciais transtornos mentais, quando comparado com prevalências descritas por Assumpção de 8,7\% para hiperatividade/desatenção, 4\% para sinais de depressão, 5,9\% para ansiedade e 3,4\% para sintomas de conduta. ${ }^{6}$ Porém, ressaltamos que o estudo foi realizado em uma amostra de pacientes acompanhados por comorbidades clínicas, que contribuiriam para o surgimento de transtornos mentais.

Quanto aos fatores significativos neste estudo $(\mathrm{p}<0,05)$ para o surgimento de transtornos mentais - doença mental dos pais, depressão materna no período até 03 meses após o nascimento da criança, e pais que não vivem juntos atualmente - a história de doença mental dos pais é fator significativo por herança genética e por influenciar nos cuidados com a criança.

O estresse durante a gravidez está associado ao aumento de partos prematuros, natimortos, depressão pós-parto, psicose puerperal, baixo peso no nascimento, problemas respiratórios, ansiedade e depressão do bebê. Uma mãe com depressão no período até 03 meses após o nascimento da criança, não fornecerá o cuidado essencial na fase de amamentação e formação do vínculo mãe-bebê. ${ }^{8}$

Pesquisa realizada com 648 crianças de um a dez anos, verificou a interação de múltiplos fatores de risco, envolvendo divórcio dos pais e crianças com transtornos psiquiátricos. Após oito anos de seguimento, as crianças apenas sob custódia materna aumentavam o risco de ansiedade, que foi mais acentuado nos meninos do que nas meninas, sem correlação com faixa etária. ${ }^{9} 10$ Que tipo de apoio pode trazer para a vida familiar a presença da figura paterna no domicílio: seria o fato de a criança crescer em famílias monoparentais que afeta sua saúde mental ou o efeito negativo do estresse pela mudança na estrutura familiar, quando o pai deixa o domicílio?

Pobreza, doença psiquiátrica materna e violência familiar foram fortemente associadas a taxas elevadas de transtornos psiquiátricos prováveis entre jovens brasileiros de 7 a 14 anos. Embora seja tentador concluir que adversidades sociais causaram problemas comportamentais nas crianças, os problemas das crianças podem ter evocado a depressão materna ou disciplina severa. ${ }^{11}$

Psicopatologias da infância já foram associadas com diversos fatores: idade jovem, menor escolaridade maternas e baixo 
QI da criança indicam pior prognóstico. Saúde física pobre está associada a má saúde mental, particularmente distúrbios emocionais. Há evidências de que desnutrição, complicações perinatais, infecções do sistema nervoso central podem desempenhar um papel. Experimentar eventos adversos da vida, como luto e divórcio dos pais, também foi encontrado como importante preditor de má saúde mental infantil. Além das variáveis individuais, fatores familiares importantes foram identificados como de risco: estresse ou doença mental nos pais, má disciplina familiar, uso de castigo físico, desarmonia parental e violência conjugal. Status socioeconômico e educação parental são inversamente associados a transtornos psiquiátricos na maioria dos estudos. Fatores escolares e da comunidade também podem ser importantes: política de tolerância zero em relação à violência nas escolas e altos níveis de capital social nas comunidades têm sido sugeridos como fatores de proteção. Foram observadas diferenças nos fatores de risco para diferentes psicopatologias: sintomas emocionais foram associados com o estresse dos pais, más condições de saúde e sexo feminino; problemas comportamentais foram associados com viver em família não tradicional, abuso de álcool na família, estresse parental e punição física severa; hiperatividade foi associada com baixo QI e sexo masculino. ${ }^{12}$

Em pesquisa de Hildebrand et al, ${ }^{12}$ em que quase metade das famílias eram monoparentais, geralmente com genitora como única responsável, a mãe era indicada como principal

\section{REFERÊNCIAS}

1. Coêlho BM, Pereira JG, Assumpção TM, Santana GL Jr. (orgs.) Psiquiatria da infância e da adolescência: guia para iniciantes. Novo Hamburgo: Sinopsys editora; 2014.

2. Rohde LA, Zavaschi ML, Lima D, Assumpção FB Jr, Barbosa $\mathrm{G}$, Golfeto $\mathrm{JH}$, et al. Quem deve tratar crianças e adolescentes com transtornos mentais? O espaço da psiquiatria da infância e adolescência em questão. Rev Bras Psiquiatr. 2000;22(1):2-3.

3. Waddell C, Offord DR, Shepherd CA, Hua JM, McEwan K. Child psychiatric epidemiology and Canadian public policy-making: the state of the science and the art of the possible. Can J Psychiatry. 2002;47(9):825-32.

4. Johnson SB, Riley AW, Granger DA, Riis J. The science of early life toxic stress for pediatric practice and advocacy. Pediatrics. 2013;131(2):319-27.

5. Dumas JE. Psicopatologia da infância e da adolescência. Porto Alegre: Artmed; 2011.

6. Assumpção FB Jr. Psiquiatria infantil brasileira: um esboço histórico. São Paulo: Lemos Editorial; 1995.

7. Goodman, R. Questionário de capacidades e dificuldades (SDQ) [Internet]. 1999 [acesso em: 2016 maio 15]. Disponível em: http:// www.sdqinfo.com/py/sdqinfo/b3.py?language $=$ Portugueseqz $(\mathrm{Braz}$ il) agressora, e a maioria das famílias apresentavam histórico de violência intrafamiliar transgeracional, ou seja, os responsáveis também foram vítimas de violência doméstica. Pode-se pressupor que assumir responsabilidades familiares, socioeconômicas e educativas de um filho, sozinha, torna-se um fator de mais vulnerabilidade da mãe, pois essa situação pode ser vivenciada por ela como ausência de apoio, principalmente quando associada ao histórico de violência doméstica dessas genitoras. Esses dados condizem com a literatura que aponta como possíveis fatores de risco para o desenvolvimento de transtornos mentais: baixa renda familiar, baixa escolaridade dos responsáveis, violência comunitária, conflitos intrafamiliares, envolvimento dos responsáveis com criminalidade, punição física severa, aspectos psicológicos dos cuidadores, o fato de ser órfão, vivenciar processo de institucionalização precoce/prolongada e condições precárias de saúde na infância e adolescência. ${ }^{13}$

Concluímos que a prevalência de transtornos mentais nas crianças e adolescentes já acompanhados por outras comorbidades clínicas é consideravelmente maior que na população geral; e que os fatores significativos para o desenvolvimento de transtornos mentais na criança estão relacionados aos pais, por contribuição genética, por patologia materna, que influencia nos primeiros cuidados ao recém-nascido, ou pela ausência paterna durante o crescimento da criança.

8. Marcelli D, Cohen D. Infância e psicopatologia. Porto Alegre: Artmed; 2010.

9. Kasen S, Cohen P, Brook JS, Hartmark C. A multiple-risk interaction model: effects of temperament and divorce on psychiatric disorders in children. J Abnorm Child Psychol. 1996;24(2):121-50.

10. Achenbach TM, Rescorla LA, Ivanova MY. International epidemiology of child and adolescent psychopathology I: diagnoses, dimensions, and conceptual issues. J Am Acad Child Adolesc Psychiatry. 2012;51(12):1261-72.

11. Fleitlich B, Goodman R. (2001). Social factors associated with child mental health problems in Brazil: cross sectional survey. British Medical Journal. 2001;323(7313):599-600.

12. Goodman A, Fleitlich-Bilyk B, Patel V, Goodman R. Child, family, school and community risk factors for poor mental health in Brazilian School children. J Am Acad Child Adolesc Psychiatry. 2007;46(4):448-56.

13. Hildebrand NA, Celeri EH, Morcillo AM, Zanolli ML. Violência doméstica e risco para problemas de saúde mental em crianças e adolescentes. Psicol Reflex Crit. 2015;28(2):213-21.

\section{Como citar:}

Ferraz IE, Leite AJ, Campos EM, Jorge IF, Espírito Santo SR, Parente GA, et al. Fatores psicossociais associados ao desenvolvimento de transtornos psiquiátricos em crianças e adolescentes. Rev Med UFC. 2017 mai-ago;57(2):8-13. 